This item is the archived peer-reviewed author-version of:

\title{
Distributed low-cost microphone array for 3D localization for bio-acoustic applications
}

\section{Reference:}

Verreycken Erik, Daems Walter, Steckel Jan.- Distributed low-cost microphone array for 3D localization for bio-acoustic applications

2017 IEEE SENSORS, October 29 - November 1, 2017, Glasgow, Scotland, UK - ISBN 978-1-5386-4056-2 - Piscataway, N.J., IEEE, 2017, p. 1-3

Full text (Publisher's DOI): http://dx.doi.org/doi:10.1109/ICSENS.2017.8234059

To cite this reference: http://hdl.handle.net/10067/1479510151162165141 


\section{Distributed Low-cost Microphone Array for 3D Localization for Bio-acoustic Applications}

\author{
Erik Verreycken \\ FTI - CoSys Lab \\ University of Antwerp \\ Antwerp, Belgium
}

\author{
Walter Daems \\ FTI - CoSys Lab \\ University of Antwerp \\ Antwerp, Belgium
}

\author{
Jan Steckel \\ FTI - CoSys Lab \\ University of Antwerp \\ Antwerp, Belgium
}

\begin{abstract}
In this paper we present a multi-scale microphone array aimed at 3D source localization, with applications in bioacoustic animal tracking. We propose a hardware architecture for wirelessly synchronized sensor nodes consisting of small 5element microphone arrays. Using this distributed sensor array we set up a probabilistic source localization problem, which we subsequently demonstrate to be able to estimate the position of a bat-like sound source in three dimensions. The proposed hardware architecture allows scalable source localization in a wide variety of application domains.
\end{abstract}

\section{INTRODUCTION}

Acoustic localization using sensor arrays has been thoroughly studied over the past decades [1]-[4]. In this paper we describe a low-cost approach for the localization of an acoustic source in 3D space using a Wireless Sensor Network (WSN) which consists of multiple sensor sub-arrays. Each individual sub-array is positioned in the environment with a known location and orientation. Many existing solutions to acoustic 3D localization of sources (e.g. animals like bats) use large-scale arrays of single-element microphones [1], [2]. The position of the acoustic source can be derived by analyzing the Time Difference of Arrival (TDOA) of the received signals at all array elements [3]. By introducing small-scale subarrays in the large-scale array, each node can first perform an estimate of the arrival angle. Several of these arrival angle estimates can lead to a 3D source position [4]. In this paper we present a probabilistic framework to combine the smallscale and large-scale TDOA measurements to perform accurate 3D localization of an acoustic source using a wirelessly synchronized sensor array. The remainder of this paper is structured as follows: in section II the probabilistic source localization algorithm is explained. Section III gives more details on our system and shows some experimental results. We discuss these results and indicate directions for future work in the final section.

\section{Probabilistic Source Localization}

The goal of source localization is to retrieve the position $\vec{P}_{s}$ of a sound source:

$$
\vec{P}_{s}=\left[x_{s}, y_{s}, z_{s}\right]^{T}
$$

To this end we use a probabilistic localization technique that results in not only a most likely position, but will retain information on the likelihood of this and other possible locations.

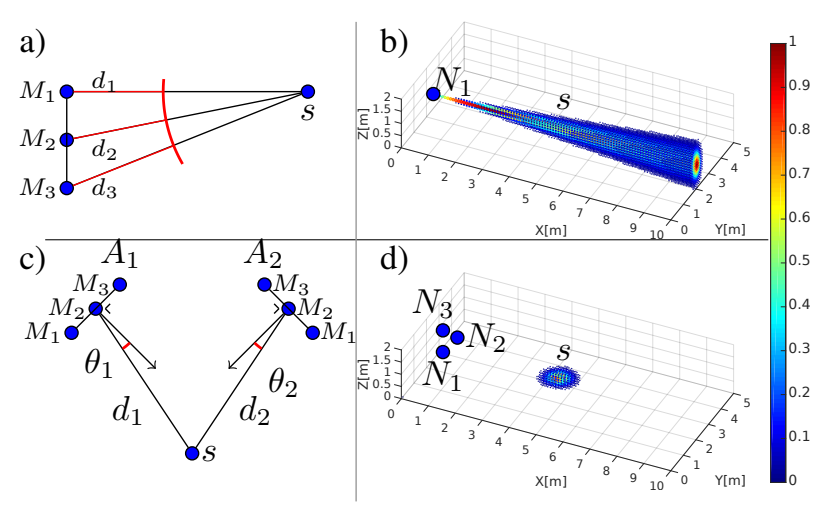

Fig. 1. Panel a) is the 2D representation of small-scale TDOA estimation on a single node (essentially Angle-of-arrival estimation). The resulting likelihood function is cone-shaped, and can be seen in panel b). Panel c) illustrates the combination of several local small-scale AOA estimates into a single 3D position estimate. The resulting likelihood function is shown in panel d). This likelihood function can be used to estimate the position of a sound source in 3D.

A microphone array can record the source's sound at different locations so that TDOA techniques can be used to locate the source. We refer to this approach as large-scale TDOA estimation of the source's position. The microphone array is divided in multiple sub-arrays consisting of several closelyspaced (orders of centimeters) microphones (fig 1a). In this way, these sub-arrays form the nodes of a larger WSN as illustrated in figure 1c. Each of these individual nodes can perform an estimate of the arrival angle of the impinging sound wave (fig 1b), which is referred to as small-scale TDOA estimation. Localization in 3D can then be performed using the entire network by combining multiple sub-arrays into a larger super-array through the combination of several Angle of Arrival (AOA) estimates. The resulting location estimate can be seen in figure $1 \mathrm{~d}$.

\section{A. Small-scale Source Localization}

Our nodes each contain a sub-array of 5 microphones and an Analog to Digital Converter (ADC) able to sample six channels synchronously. Since the data-acquisition on a single node is done using a simultaneous sampling ADC we assume the individual microphone recordings to be accurately 
synchronized. When a sound is received, the relative arrival times can be calculated (for example through generalized cross correlation [5]) and a local measurement vector $\vec{M}_{L}$ can be constructed by comparing differences in arrival time between all microphone pairs. To keep the formulas below compact, we restricted ourselves to 3 microphones:

$$
\vec{M}_{L}=\left[d_{1}-d_{2}, d_{1}-d_{3}, d_{2}-d_{3}\right]^{T}
$$

with $d_{j}$ the relative arrival time for microphone $j$. Using the individual location and orientation information of each sensor node, we can calculate $\vec{M}_{L}$ for each source location $\vec{P}_{s}$. This prediction is used to construct a distance vector $\vec{D}\left(\vec{P}_{s}\right)$ as a function of $\vec{P}_{s}$ :

$$
\vec{D}=\left[d_{1}-d_{2}, d_{1}-d_{3}, d_{2}-d_{3}\right]^{T}
$$

The likelihood $\mathcal{L}_{L}$ of the measurement vector $\vec{M}_{L}$ given a proposed source position $\vec{P}_{s}$ can be expressed for each node $i$ as:

$$
\mathcal{L}_{L, i}\left(\vec{M}_{L} \mid \vec{P}_{s}\right)=\exp \left[-\left(\vec{M}_{L, i}-\vec{D}\right)^{T} \cdot \Sigma_{L}^{-1} \cdot\left(\vec{M}_{L, i}-\vec{D}\right)\right]
$$

with $\Sigma_{L}$ the expected covariance matrix of the measurement errors. By multiplying the likelihoods of each microphone pair together we get a combined likelihood estimate for the measurement over all nodes in the array:

$$
\mathcal{L}_{L}\left(\vec{M}_{L} \mid \vec{P}_{s}\right)=\prod_{i=1}^{n} \mathcal{L}_{L, i}\left(\vec{M}_{L} \mid \vec{P}_{s}\right)
$$

Bayes' theorem can then be used to assess the posterior distribution probability $\mathcal{P}$ of each position $\vec{P}_{s}$ given the measurement $\vec{M}_{L}$ :

$$
\mathcal{P}\left(\vec{P}_{s} \mid \vec{M}_{L}\right)=\frac{\mathcal{L}_{L}\left(\vec{M}_{L} \mid \vec{P}_{s}\right) \cdot \mathcal{P}\left(\vec{P}_{s}\right)}{\mathcal{P}\left(\vec{M}_{L}\right)}
$$

with $\mathcal{P}\left(\vec{P}_{s}\right)$ the prior distribution for position $\vec{P}_{s}$ and $\mathcal{P}\left(\vec{M}_{L}\right)$ the marginal of the posterior distribution. Since the microphones of a single node are spaced relatively close together in a single plane, $\mathcal{L}_{L, i}\left(\vec{M}_{L} \mid \vec{P}_{s}\right)$ does not result in a unimodal shape around the true source position, but rather in a cone shape where all 3D points that have equal azimuth and elevation with respect to the local node have an equal likelihood, which can be interpreted as an estimate of the arrival angle. In other words, the likelihood $\mathcal{L}_{L}$ is range independent with respect to the node's spherical coordinate system (see figure 1b). The product of all the individual likelihoods, $\mathcal{L}_{L}\left(\vec{M}_{L} \mid \vec{P}_{s}\right)$, will have a (unimodal) peak around the true source position in the case of Gaussian measurement errors (see figure 1d).

\section{B. TDOA between Nodes}

At network scale, multiple sub-arrays are combined into a larger WSN, which we have previously referred to as largescale TDOA estimation. One or more channels from each sub-array are combined and the TDOA can be calculated. At this point it is quintessential that the individual subarrays are accurately synchronized. In previous work [6] we described how the simultaneous generation of pseudorandom numbers across different nodes can be used for post measurement synchronization using cross correlation of the pseudo-random signal. The synchronization is performed in a distributed network of pulse-coupled oscillators inspired by the synchronization approach followed by many species of fireflies. A global measurement vector $\vec{M}_{G}$ is constructed as follows:

$$
\vec{M}_{G}=\left[d_{1}-d_{2}, d_{1}-d_{3}, d_{2}-d_{3}\right]^{T}
$$

where $d_{i}$ can be any microphone of any sub-array. Again, for notational clarity, we restricted ourselves to 3 microphones. Similar to the small-scale TDOA approach, we can create a vector $\vec{D}_{G}$ for each source position $\vec{P}_{s}$, allowing us to calculate the likelihood $\mathcal{L}_{G}$ for measurement $\vec{M}_{G}$, given $\vec{P}_{s}$ :

$$
\mathcal{L}_{G}\left(\vec{M}_{G} \mid \vec{P}_{s}\right)=\exp \left[-\left(\vec{M}_{G}-\vec{D}_{G}\right)^{T} \cdot \Sigma_{G}^{-1} \cdot\left(\vec{M}_{G}-\vec{D}_{G}\right)\right]
$$

As the large-scale array has a much larger size than the local curvature of the arriving wavefronts, the probability of any point $\vec{P}_{s}$ being the point of origin of the sound then becomes:

$$
\mathcal{P}\left(\vec{P}_{s} \mid \vec{M}_{G}\right)=\frac{\mathcal{L}_{G}\left(\vec{M}_{G} \mid \vec{P}_{s}\right) \cdot \mathcal{P}\left(\vec{P}_{s}\right)}{\mathcal{P}\left(\vec{M}_{G}\right)}
$$

This posterior distribution has a unimodal peak around the true source position in case of unbiassed Gaussian measurement errors.

\section{Combining Local and Global Measurements}

Having obtained a posterior position estimate for the source in two separate ways (small-scale and large-scale TDOA), we can combine both posterior distributions into a single distribution for the source position:

$$
\mathcal{P}\left(\vec{P}_{s} \mid \vec{M}\right)=\frac{\mathcal{P}\left(\vec{P}_{s} \mid \vec{M}_{G}\right) \cdot \mathcal{P}\left(\vec{P}_{s} \mid \vec{M}_{L}\right)}{\sum_{\forall \vec{P}_{s}}\left(\mathcal{P}\left(\vec{P}_{s} \mid \vec{M}_{G}\right) \cdot \mathcal{P}\left(\vec{P}_{s} \mid \vec{M}_{L}\right)\right)}
$$

In a single-measurement source estimation problem, the best guess for the sound location can be found as:

$$
\widetilde{P}_{s}=\operatorname{argmax} \mathcal{P}\left(\vec{P}_{s} \mid \vec{M}\right)
$$

which can be solved using a non-linear constrained maximization algorithm. Alternatively, in a path-tracking application, the posterior distribution can be fed into a particle filter together with a motion model of the source that is to be tracked to arrive at a recursive path-tracking solution. In this paper however we will focus on single-snapshot localization experiments.

\section{HARDWARE PROTOTYPE AND EXPERIMENTAL RESULTS}

To demonstrate the efficacy of the proposed localization approach we designed and developed a Printed Circuit Board (PCB) with a 5 microphone sub-array as seen in figure 2 a. All 5 microphones are sampled at a sample rate of $285 \mathrm{kHz}$ by a 6-channel simultaneous sampling ADC which ensures that the local microphone data is synchronized. When a recording is made we use a matched filter to determine if and when a 


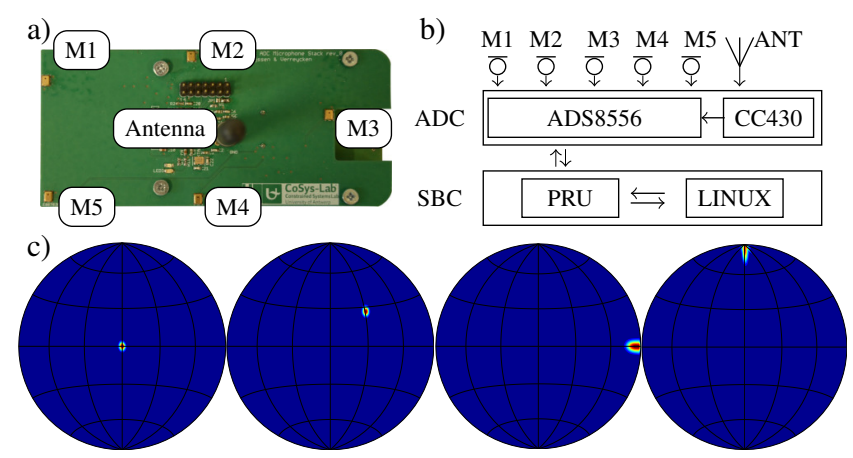

Fig. 2. Panel a): Our single-board microphone sub-array. This PCB contains 5 ultrasonic microphones placed at the edges of the board and a $433 \mathrm{MHz}$ antenna for wireless synchronization. The PCB stacks on top an ADC module and a SBC (Single Board Computer). Panel b): Overview of the hardware stack. The SBC at the bottom manages digital communication with the ADC above i.e. data offload and when to start or stop a capture. The ADC only receives analog data from the microphone array. The synchronization signal is sent as a 6th analog channel to the ADC. Panel c: Experimental AOA estimates made by a single sub-array in an office-like environment. The likelihood function $\mathcal{L}_{L, i}\left(\vec{M} \mid \vec{P}_{s}\right)$, sampled on a hemisphere, is plotted using a Lambert Equal Area projection. Gridlines spaced at $30^{\circ}$, contour lines spaced at $10 \%$ of the maximum probability.

certain reference sound (e.g. a prerecorded bat call) is present in the measurement. The 6th channel of the ADC is reserved as a synchronization channel to record the XOR-ed state of the synchronized pseudorandom generator (see [6] for details). The RF-based synchronization is performed over a $433 \mathrm{MHz}$ communication link based on a Texas Instruments CC430 RFSoC through the SimpliciTI communication protocol.

As shown in figure $2 \mathrm{~b}$ we built a hardware stack with on top the microphone array. The array stacks on top of the ADC board, which sits on top of a Single Board Computer (SBC), in this case a BeagleBone Black (BBB). The BBB runs a general purpose Linux OS and is equipped with a Programmable RealTime Unit (PRU). The PRU is a $200 \mathrm{MHz}, 32$ bit real-time core that can be programmed directly in assembler and guarantees single-clock IO operations. Therefore, the PRU is ideally suited to perform timing-critical tasks such as interfacing with a high-speed ADC, as the control of this ADC is very timing sensitive.

We placed our node in a room together with an ultrasonic speaker. The speaker emits an imitation of a bat call, i.e. a frequency sweep from $60 \mathrm{kHz}$ to $20 \mathrm{kHz}$ in $3 \mathrm{~ms}$ [7]. As explained in section II-A a single node can estimate the AOA in azimuth and elevation. Figure $2 \mathrm{c}$ shows the likelihood $\mathcal{L}_{L, i}\left(\vec{M} \mid \vec{P}_{s}\right)$ of a single measurement for a single node. The points $\vec{P}_{s}$ were distributed uniformly over a hemisphere with a radius of 5 meters, allowing the creation of the Lambert Equal Area Projections of the likelihood function.

To illustrate the full 3D localization of a sound source, we simulated an array consisting of 3 nodes, with each node being identical to the nodes described in the previous section. We placed the nodes in an array as shown in figure 1c. We simulated an acoustic source following a typical trawlingbat flight path (e.g. Myotis daubentonii). The snapshot-based

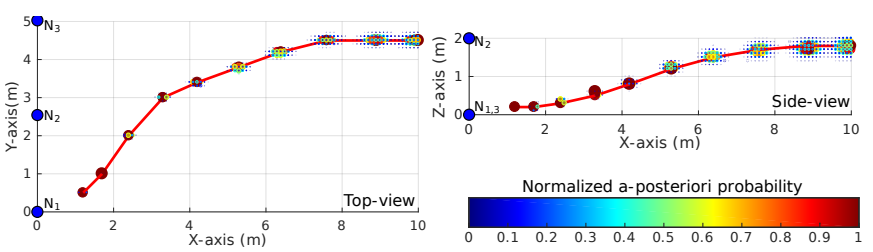

Fig. 3. Tracking a typical simulated flight path of a trawling bat like Myotis daubentonii. The red line is the true flight path, the red dots indicate the estimated position. The remaining colored dots illustrate the width of the a-posteriori probability function of each position estimate. The error in Euclidean distance between our best guess and the actual source position ranges between $5 \mathrm{~mm}$ and $19 \mathrm{~cm}$ with a mean of $5 \mathrm{~cm}$. The nodes lie on a single plane at $x=0$, their locations have been marked in blue.

localization algorithm is capable of reconstructing the sources flight-path as shown in figure 3.

\section{CONCLUSION AND FUTURE WORK}

In this paper we introduced a multi-scale microphone array for 3D sound source localization aimed at bioacoustics. We proposed a probabilistic framework in which a source can be localized in $3 \mathrm{D}$ through a combination of small-scale TDOA (i.e. the implicit combination of multiple AOA estimates) and large-scale TDOA. We introduced a hardware architecture which supports this distributed localization technique through a synchronized wireless sensor network, and demonstrated the feasibility of the proposed localization algorithm for bioacoustic tracking applications. As future work, we plan to introduce the probabilistic formulation of the source's position in a recursive Bayesian filter to accurately track the path of the source in three dimensions over time.

\section{ACKNOWLEDGEMENTS}

$\mathrm{EV}$ is funded by a grant from the special research fund of the University of Antwerp.

\section{REFERENCES}

[1] G. Simon, M. Maroti, A. Ledeczi, G. Balogh, B. Kusy, A. Nadas, G. Pap, J. Sallai, and K. Frampton, "Sensor network-based countersniper system," in Proceedings of the 2nd international conference on Embedded networked sensor systems - SenSys '04. New York, New York, USA: ACM Press, 2004, p. 1.

[2] K. Motoi, M. Sumiya, E. Fujioka, and S. Hiryu, "Three-dimensional sonar beam-width expansion by Japanese house bats ( Pipistrellus abramus ) during natural foraging," The Journal of the Acoustical Society of America, vol. 141, no. 5, pp. EL439-EL444, may 2017.

[3] J. Sallai, W. Hedgecock, P. Volgyesi, A. Nadas, G. Balogh, and A. Ledeczi, "Weapon classification and shooter localization using distributed multichannel acoustic sensors," Journal of Systems Architecture, vol. 57, no. 10 , pp. 869-885, nov 2011.

[4] S. D. Holland, R. Roberts, D. Chimenti, and J. H. Song, "An ultrasonic array sensor for spacecraft leak direction finding," Ultrasonics, vol. 45, no. 1-4, pp. 121-126, dec 2006.

[5] C. Knapp and G. Carter, "The generalized correlation method for estimation of time delay," IEEE Transactions on Acoustics, Speech, and Signal Processing, vol. 24, no. 4, pp. 320-327, aug 1976.

[6] E. Verreycken, D. Laurijssen, W. Daems, and J. Steckel, "Firefly based distributed synchronization in Wireless Sensor Networks for passive acoustic localization," in 2016 International Conference on Indoor Positioning and Indoor Navigation (IPIN). IEEE, oct 2016.

[7] T. Andreassen, A. Surlykke, J. Hallam, and D. Brandt, "Ultrasonic recording system without intrinsic limits," The Journal of the Acoustical Society of America, vol. 133, no. 6, pp. 4008-4018, jun 2013. 